\title{
GroupCast: Preference-Aware Cooperative Video Streaming With Scalable Video Coding
}

\author{
Anis Elgabli, Muhamad Felemban, Student Member, IEEE, and Vaneet Aggarwal, Senior Member, IEEE
}

\begin{abstract}
In this paper, we propose a preference-aware cooperative video streaming system for videos encoded using scalable video coding (SVC). In the proposed system, the collaborating users are interested in watching a video together on a shared screen. However, the willingness of each user to cooperate is subject to her own constraints such as user data plans. Using SVC, videos are segmented into chunks and each chunk is encoded using layers, where each layer can be fetched through any of the collaborating users. We formulate the problem of finding the optimal quality decisions and fetching policy of the SVC layers of video chunks subject to the available bandwidth, chunk deadlines, and cooperation willingness of the different users as an optimization problem. The objective is to optimize a QoE metric that maintains a trade-off between maximizing the playback rate of every chunk and ensuring fairness among all chunks to achieve the minimum skip (stall) duration without violating any of the imposed constraints. We propose an offline algorithm to solve the non-convex optimization problem when the bandwidth prediction is non-causally known. This algorithm has a run-time complexity that is polynomial in the video length and the number of collaborating users. Furthermore, we propose an online version of the algorithm for practical scenarios, where erroneous bandwidth prediction for a short window is used. Real implementation with android devices using SVC encoded video and a public dataset of bandwidth traces reveals the robustness and performance of the proposed algorithm and shows that the algorithm significantly outperforms round robin-based mechanisms in terms of avoiding skips/stalls and fetching video chunks at their highest quality possible.
\end{abstract}

Index Terms-Cooperative video streaming, scalable video coding, rate adaptation, non convex optimization.

\section{INTRODUCTION}

$\mathbf{V}$ IDEO streaming is the dominant contributor to the cellular network traffic. Currently, video content accounts for $50 \%$ of cellular network traffic and it is expected to account for around $75 \%$ of the mobile data traffic by This work was supported in part by the U.S. National Science Foundation under Grant CCF-1527486 and Grant CNS-1618335. This paper was presented, in part, at the IEEE Infocom Workshop 2018 [1]. (Corresponding author: Vaneet

Aggarwal.

A. Elgabli was with the School of ECE, Purdue University, West Lafayette, IN 47907 USA. He is now with the Center of Wireless Communications, University of Oulu, 90014 Oulu, Finland (e-mail: aelgabli@purdue.edu).

M. Felemban was with the School of ECE, Purdue University, West Lafayette, IN 47907 USA. He is now with KFUPM, Dhahran 31261, Saudi Arabia (e-mail: mfelemban@kfupm.edu.sa).

V. Aggarwal is with the School of IE, Purdue University, West Lafayette, IN 47907 USA (e-mail: vaneet@purdue.edu).

This paper has supplementary downloadable material available at http://ieeexplore.ieee.org, provided by the authors. This includes a PDF containing Appendixes A-D.
2020 [2]. This increase has forced service providers to enhance their infrastructures to support high-quality video streaming. Despite these efforts, users frequently experience low Quality-of-Experience (QoE) metrics such as choppy videos and playback stalls [3]. One prominent approach to improve the QoE is the use of cooperative video streaming technology [4], which allows to aggregate the available bandwidth for different users in order to increase the download rate; and hence increase the video playback rate. Several challenges have to be addressed when using cooperative video streaming, including finding a fetching policy among the users while using their resources efficiently.

In Scalable Video Coding (SVC), videos are segmented into chunks and each chunk is encoded into ordered layers: a base layer $(\mathrm{BL})$ and multiple enhancement layers $\left(E_{1}, \ldots, E_{N}\right)$. The base layer represents the lowest playable quality of the video, while each enhancement layer further improves the quality [5]. In order to play a video chunk at the $i^{\text {th }}$ enhancement layer, all layers from 0 to $i$ have to be downloaded. Consequently, adaptive SVC streaming can allow playback at the lowest quality if the enhancement layers of a chunk have not been fetched before its deadline. In cooperative video streaming, multiple layers of the same chunk can be simultaneously fetched by collaborating users.

To motivate our problem, consider a scenario where a group of users are in a remote location with poor Internet coverage, e.g., a camp site. The users are interested in watching a football game on a High-Definition screen. The users are willing to collaborate in streaming the game using their mobile phones, which can operate on multiple carriers, e.g, AT\&T, Verizon and, T-Mobile, and have varying data plan contracts, e.g., an unlimited, a 6GB, or a $3 \mathrm{~GB}$ plan. Such scenario can lead to a diversity in the users channels and thus an improvement in video quality over the aggregated channel.

Even when all the phones operate on the same carrier, the aggregated channel allows to improve the quality of the video. To illustrate, assume that there are $N$ collaborating users (willing to cooperate and watch the same video) who are connected to a single base station and competing over the available resources with $M$ other users who are connected to the same base station. Assuming equivalent channel strength, the collaborating users use $\frac{N}{M+N}$ of the base station resources for streaming the video. For example, the fraction of the resources used by two and three collaborating users is $\frac{2}{10}$ and $\frac{3}{11}$ respectively when there are 8 other users served by the same base station. Therefore, the percentage of the resources used by the collaborating users increases with $N$. 


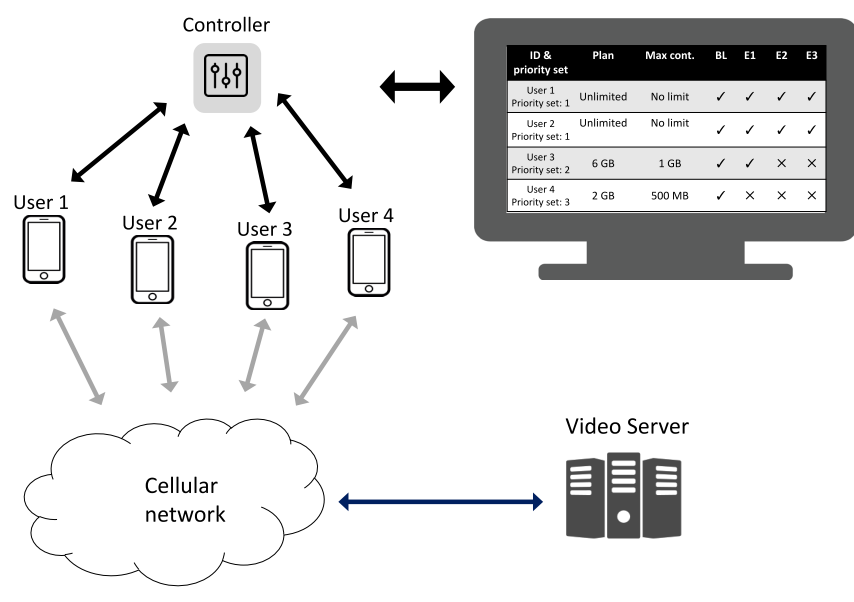

Fig. 1. Example to illustrate the different inputs by the different users. These inputs include the priority set, amount of data they can contribute, and the different layers they can help fetch to maximize the quality of experience for video viewing.

We assume that the bottleneck is the wireless cellular link, rather than the backbone network. Furthermore, we assume users impose maximum contribution limit based on their remaining data. The maximum contribution indicates the maximum amount of data a user can participate in downloading for the entire video. For example, maximum contribution can be specified as a percentage of the remaining data in the plan. Moreover, users can specify the highest quality up to which they can help downloading. Based on the users' imposed constraints, they are assigned to multiple priority sets, where users belong to a higher priority set can help in fetching more layers of the video.

Figure 1 shows an illustrative example for a setup of four users. The video is encoded into four layers, a base layer and three enhancement layers. Users 1 and 2 have unlimited data plans and have no maximum contribution limits. The two users are assigned to the top priority set, i.e., priority set 1 and, consequently, can fetch all layers. On the other hand, user 3 has a limited data plan (6 GB/month). User 3 is assigned to the second priority set and can contribute to increase the quality of the chunks by downloading up to the $1^{\text {st }}$ enhancement layer if the first two users failed to do so. Although User 3 helps in fetching chunks, this user also has a maximum contribution limit of $1 \mathrm{~GB}$. User 4 is assigned to the third priority set and can help in obtaining only base layers of chunks when the other users are not able to obtain them. Further, the maximum contribution of user 4 is $500 \mathrm{MB}$.

In this paper, we propose GroupCast, a novel preferenceaware cooperative video streaming system that allows multiple collaborative users to watch SVC-encoded videos with at high quality on a shared screen. In particular, we propose algorithms for two classes of streaming scenarios: skip based and no-skip based streaming. In both classes, each chunk is associated with a playback deadline. In the skip based streaming (realtime streaming), the chunks that are not received before their deadlines are skipped. On the other hand, in no-skip based streaming, if a chunk cannot be fully downloaded by its initial deadline, this chunk is not skipped; instead, a stall (re-buffering) will take place until the chunk is fully downloaded. Therefore, in skip based streaming, the user will still experience a stall at the playback time corresponding to the skipped chunk, but subsequent chunks meet their deadlines. On the other hand, in no-skip based streaming (Video on demand), we do not skip chunks. Every chunk is played, but quality decisions are made such that the stall (re-buffering) duration is at its minimum, and whenever, there is a stall, we shift the deadlines of all chunks after this stall by a time equal to the stall duration. Although the system and the problem formulation discussed in the paper are described for SVC, they are applicable to any layered video coding technique. For example, videos encoded using Scalable High efficiency Video Coding (SHVC), which is a scalable extension of H.265/HEVC [6], can be cooperatively streamed using the proposed approach.

An optimization problem is formulated when the bandwidth can be obtained offline for both the scenarios. The optimization problem considers a QoE metric that maximizes a concave utility function of the chunk qualities and minimizes skip/stall duration while respecting the priority of the users (users belong to lower priority sets should be used less) and their imposed maximum contributions. Since the proposed optimization problem is non-convex, optimal low-complexity algorithms are not feasible. Therefore, we provide efficient algorithms that have a polynomial run-time complexity with respect to the number of contributing users and fetched layers. Moreover, we provide some guarantees of the proposed algorithm.

The proposed algorithms are further extended to the practical online case where the bandwidth is only known for a short time ahead and can be erroneous. Any bandwidth prediction method like the crowd-sourcing, or harmonic mean prediction method [7], [8], can be used which provides the bandwidth prediction with some errors. The proposed algorithms are implemented and tested using Android devices with SVC encoded videos and real bandwidth traces. The algorithms are shown to outperform the considered baselines significantly. The contributions of this paper are summarized as follows.

- We propose a cooperative video streaming system, called GroupCast, that enables collaborative users to stream SVCencoded videos.

- We provide polynomial complexity algorithms to solve the proposed optimization problem, and we provide theoretical guarantees of the proposed offline algorithm. Moreover, we propose an online window-based algorithms for online scenario in which the bandwidth can be predicted for short time ahead with prediction error. Moreover, different algorithms are proposed for the skip based and the no-skip based streaming scenarios.

- We evaluate the proposed algorithms with a real implementation using Android devices, SVC-encoded videos, and public bandwidth traces. The results show that GroupCast significantly outperform round robin based schemes.

The rest of the paper is organized as follows. Section II discusses related work. Section III describes the system model. Section IV describes the problem formulation for skip 
based cooperative video streaming. In section $\mathrm{V}$, a set of polynomial-time algorithms are provided for this non-convex problem. No-skip scenario is discussed in section VI. Section VII presents the trace-driven evaluation results with comparison to different baselines. Section VIII concludes the paper.

\section{RELATED WORK}

Adaptive Bit Rate (ABR) Streaming for Single User. Commercial systems such as Apple's HLS [9], Microsoft's Smooth Streaming [10], and Adobe's HDS [11] are different variants of ABR streaming. Various approaches for making ABR streaming decisions have been investigated, including control theory [7], [12], Markov Decision Process [13], machine learning [14], client buffer information [15], and data-driven techniques [16]-[18].

In a single user video streaming scenario, the rate adaptation techniques can be classified into: (i) buffer-based rate adaptation techniques, in which the quality decisions of the next chunks to fetch is decided based on the current buffer occupancy (e.g., [15]), (ii) prediction-based techniques in which a prediction method such as crowd-sourcing [19], [20] or harmonic mean [7] is used to predict the bandwidth of the next few seconds and decide the quality of the next chunks to fetch, and (iii) a combination of buffer and prediction in which the current buffer occupancy and the predicted bandwidth are incorporated in order to decide the quality of the next few chunks [7], [21]. The third class is shown to outperform the first two classes [7], [21]. In our proposed formulation, we incorporate both buffer and prediction into our quality decisions.

Adaptive SVC Streaming for Single User. SVC received the final approval to be standardized as an amendment of the H.264/MPEG-4 AVC (Advanced Video Coding) standard in 2007 [22]. The first dataset and toolchain for SVC is published in [23]. Authors in [24], [25] have proposed new rate adaptation algorithms for SVC that alternate between prefetching future base layers and backfilling current enhancement layers. In [26], an optimization based rate adaptation technique for single link SVC-encoded videos is proposed. In this paper, the objective is to optimize a QoE metric that maintains a tradeoff between maximizing the quality of each chunk and ensuring fairness among all chunks for the minimum stall/skip duration.

Multi-Path Video Streaming. Multi-path TCP (MPTCP [27]) is proposed for multi-path streaming in which a single client is opening parallel connections with a server using orthogonal links, i.e., LTE and WiFi. However, MPTCP does not work across users and have several implementation and security issues [8], [28]. The authors of [8] proposed a heuristic approach for Multi-Path rate adaptation in which multiple servers, a single client with two links (LTE and WiFi), and AVC encoded videos are considered. However, this rate adaptation technique considers only two paths, and is proposed for ABR based streaming. Thus, it does not exploit the flexibility of SVC where a fine-grained decision per layer can be taken. Furthermore, these work do not account for maximum contribution limit on one or both of the paths. Finally, [29] considered SVC multipath preference aware streaming for SVC. However, authors consider only 2 links without imposing maximum contribution per link.

Cooperative Video Streaming. For multiple users interested in watching the same video, streaming algorithms using device-to-device (D2D) connections have been studied. In [30], authors proposed a Peer-to-Peer (P2P)-TV system that enables multiple contributors to download a single rate encoded video in which every contributor is assigned a chunk to fetch such that all chunks are retrieved before their deadlines. In [31], authors proposed network coding based cooperative video streaming for a single rate encoded video where multiple users can aggregate their bandwidth in a reliable way and fetch the video. However, none of these efforts consider SVC-encoded videos in which each chunk is available at base layer and its quality can be further improved by downloading enhancement layers.

The authors of [32] explained the role of Content-Aware Networking (CAN) to optimize network resource utilization while maintaining high QoE and QoS. The functionality of CAN is provided by enhanced network nodes, termed mediaaware network elements (MANEs), that feature virtualization support, content awareness, media processing, buffering and caching. The proposed MANEs take advantage of SVC technology in order to provide scalable streaming for users. Although the paper has laid out the main challenges of CAN in several use cases including P2P, the paper does not provide streaming algorithms for SVC-encoded videos. In [33], authors have proposed piece-picking algorithms to download selective pieces of distributed layered content over P2P networks before their deadlines. The proposed algorithms for skip-based streaming are provided based on Knapsack problem. However, the setup in [33] obtains content at a receiver from multiple peers while in our problem, multiple users obtain content simultaneously to display on a single screen.

In [34], authors have considered cooperative SVC video streaming for SVC-encoded videos but with only two layers for LTE/802.11p Vehicle-to-Infrastructure Communications. In [35], authors proposed a video assignment strategy based on the deficit round robin (DRR) for combined SVC and Multiple Description Coding (MDC), termed Co-SVC-MDCBased Cooperative video Streaming Over Vehicular Networks. In this strategy, the decision of assigning layers to users follow simple round robin strategy and does not take into account users's preferences. The authors of [36] proposed an SVC cooperative video streaming for vehicular networks when there is only one relay node per receiver, i.e., at most two contributors. In contrast to these work, we propose a generic system that can work with any number of collaborating users and any number of layers. In addition, we assume preference aware streaming from users with a maximum contribution limit per user. Moreover, we consider both skip and no-skip based streaming. The closest work to this is our prior work [37], which does not have preference awareness for different class of users, and only considers no-skip based streaming. 


\section{SySTEM MODEL}

In this section, we describe the system model. A summary table for all the symbols used throughout the paper is listed in Supplementary Material. We assume that there are $U$ users and that the SVC-encoded video is divided into $C$ chunks, where each chunk is of length $L$ seconds. The video is encoded into a base layer with rate $r_{0}$, and $N$ enhancement layers $\left(E_{1}, \cdots, E_{N}\right)$ with rates $r_{1}, \cdots, r_{N}$, respectively. Let $Y_{n}=$ $L * r_{n}$ be the size of the $n^{t h}$ layer, where $n \in\{0, \ldots, N\}$.

The users are divided into $K$ disjoint sets, where $K \leq N+$ 1. The number of users in the $k^{t h}$ set is denoted by $U_{k}$, where $k \in\{1, \cdots K\}$. Notice that $U=\sum_{k=1}^{K} U_{k}$. We denote the maximum layer that users in the $k^{t h}$ set can fetch as $N_{k}$, where $N_{k} \in\{0, \cdots, N\}$. We assume that the maximum layer that can be fetched by the $(k+1)^{t h}$ set is less than the maximum layer that can be fetched by the $k^{t h}$ set, i.e., $N_{1}>N_{2}>$ $\cdots>N_{K}$. To illustrate, assume that $K=2$, i.e., the users are divided into two sets. Therefore, if the users in the first set, i.e., $k=1$, can fetch, for example, up to the $3^{\text {rd }}$ layer, i.e., $N_{1}=3$, then users in the second set, i.e., $k=2$, can fetch up to at most the $2^{\text {nd }}$ layer, i.e., $N_{2}=2$.

We assume that each user $u$ in the $k^{\text {th }}$ set has a maximum download contribution of $\eta_{u}^{k}$. Furthermore, we assume a strict priority among users in different sets. In essence, a user in the $k^{t h}$ set fetches chunks at layers from $\left\{0, \cdots, N_{k}\right\}$ only if the users in the higher priority sets, i.e., sets 1 to $k-1$, are unable to fetch layers from $\left\{0, \cdots, N_{k}\right\}$ without violating the deadlines. In the rest of this paper, we use the terms user and link interchangeably.

The packing of the users into sets can be done automatically based on the remaining data in the plan. The remaining data can be quantized to get the set assignment. Then, a particular percentage of the available data plan can be chosen as the maximum contribution limit. Even though the set assignments and the contribution limits can be set automatically, the users have the flexibility to modify these limits. The designer may, however, limit the possibility of decreasing the priority level or the maximum contribution limit to avoid greedy behavior of certain users. However, the decrease of the maximum contribution based on available battery charge at the user may be allowable. In this paper, we assume that the user priority levels and the maximum contribution limits are known.

We consider two streaming scenarios: the skip based streaming and the no-skip based streaming. For the skip based streaming, the video is played with an initial start-up, i.e., buffering delay of $s$ seconds. There is a playback deadline for each of the chunks, where chunk $i$ needs to be downloaded by deadline $(i)=s+(i-1) L$ seconds. The chunks that cannot be received before their respective deadlines are skipped. For the no-skip based streaming, it also has a start-up delay of $s$ seconds. However, if a chunk cannot be downloaded before its deadline, it will not be skipped. Instead, a stall, i.e., re-buffering, will take place. As a result, the video will pause until the chunk is fully downloaded. Let the total stall duration from the start until the play-time of chunk $i$ be $d(i)$. Consequently, the deadline of chunk $i$ is deadline $(i)=(i-1) L+s+d(i)$. In both scenarios, the objective of the scheduling algorithm is to determine which layers need to be fetched for each chunk such that the skip/stall duration is minimized and the overall playback bitrate is maximized.

We assume that all time units are discrete and that the discretization time unit is 1 second. Let $Z_{n, i}$ denotes how much of the $n^{\text {th }}$ layer of chunk $i$ that can be fetched, i.e., if the $n^{\text {th }}$ layer of chunk $i$ can be fetched, then $Z_{n, i}=Y_{n}$; otherwise $Z_{n, i}=0$. Let $z_{n, u}^{(k)}(i, j)$ be the amount of the $n^{t h}$ layer of chunk $i$ that is fetched at time slot $j$ from the link $L_{k, u}$, where $L_{k, u}$ represents the $u^{t h}$ user in $k^{t h}$ set. The sum of $z_{n, u}^{(k)}(i, j)$ over all $u, k, j$ is $Z_{n, i}$. Let $D_{n, u}^{(k)}(i)=\sum_{j=1}^{\text {deadline }(C)} z_{n, u}^{(k)}(i, j)$ be the total amount of the $n^{\text {th }}$ layer of chunk $i$ that is fetched by $L_{k, u}$. Further, let $G_{n}^{(k)}$ be the total amount of the $n^{\text {th }}$ layer fetched from all users in the $k^{\text {th }}$ set. Thus, $G_{n}^{(k)}=\sum_{u=1}^{U_{k}} \sum_{i=1}^{C} D_{n, u}^{(k)}(i)$. We assume that at most one link can be used to get the $n^{t h}$ layer of chunk $i$. Therefore, for each layer $n$ of chunk $i$, there will be at most one link such that $D_{n, u}^{(k)}(i)>0$. Let $B_{u}^{(k)}(j)$ be the available bandwidth of the $u^{t h}$ link in the $k^{t h}$ set.

\section{Skip Based Streaming: Problem Formulation}

In this section, we present the problem formulation for the skip based scenario. The key objectives of the problem are to $(i)$ minimize the number of skipped chunks, (ii) maximize the playback rate of the video, and (iii) minimize the quality changes between the neighboring chunks. As a result, the obtained optimal fetching policy is the one that minimizes the total number of skips as the highest priority, while preferring chunks at the $n^{\text {th }}$ layer quality level over increasing the quality of some chunks to higher layers at the cost of dropping the quality of other chunks. Such strategy avoids unnecessary layer switchings and leads to a smoother and more eye-pleasant playback of the video. We first describe the proposed problem formulation of the offline version that assumes perfect prediction for the entire period of the video and infinite buffer capacity. These assumptions are relaxed when we describe the online algorithm in Section V-C.

In order to account for the three objectives with their priority orders, we maximize a weighted sum of layer sizes fetched by different users, where lower layers and users belong to higher priority sets are given higher weights. In order to do so, we introduce two-dimensional weights $\lambda_{n}^{k}$ that need to satisfy the following condition: for any users sets $k$, $k^{\prime} \in\{(k+1) \ldots K\}$, and $k^{\prime \prime} \in\{1 \ldots k\}$

$$
\lambda_{a}^{(k)} Y_{a}>C \cdot\left(\sum_{n=a}^{N} \lambda_{n}^{k^{\prime}} Y_{n}+\sum_{n=a+1}^{N} \lambda_{n}^{k^{\prime \prime}} Y_{n}\right) .
$$

The choice of weightes that satisfy Equation 1 implies two requirements. First, it implies that, for any layer $a$, the layers higher than $a$ have lower utility than a chunk at layer $a$. In the case when $a=0$, the choice of $\lambda$ implies that all the enhancement layers achieve less utility than one chunk at the base layer. The use of $\lambda$ then helps in giving higher priority to fetch more chunks at the $n^{\text {th }}$ layer quality over fetching some at higher quality at the cost of dropping the quality of other 
chunks to below the $n^{\text {th }}$ layer quality. Second, the choice of $\lambda$ implies that the highest utility that can be achieved for every layer is when it is fetched by a user belong to the set with the highest priority level. This will obey the priority order, and it will not use a lower priority user to fetch a layer that can be fetched by a higher priority user.

Accordingly, we formulate the objective function as the following.

$$
\sum_{k=1}^{K} \sum_{n=0}^{N} \lambda_{n}^{(k)} G_{n}^{(k)} .
$$

This objective is constrained by a set of constraints such as bandwidth, deadline, users maximum contribution, users-set assignment, and video decoding constraints. Accordingly, the proposed optimization problem can be formulated as follows.

$$
\text { Maximize }: \sum_{k=1}^{K} \sum_{n=0}^{N} \lambda_{n}^{(k)} G_{n}^{(k)}
$$

subject to

$$
\begin{aligned}
G_{n}^{(k)} & =\sum_{u=1}^{U_{k}} \sum_{i=1}^{C} D_{n, u}^{(k)}(i) \quad \forall n, k \\
D_{n, u}^{(k)}(i) & =\sum_{j=1}^{(i-1) L+s} z_{n, u}^{(k)}(i, j) \quad \forall i, n, k, u \\
\sum_{k=1}^{K} \sum_{u=1}^{U_{k}} D_{n, u}^{(k)}(i) & =Z_{n, i} \quad \forall i, n \\
Z_{n, i} & \leq \frac{Y_{n}}{Y_{n-1}} Z_{n-1, i} \quad \forall i, n>0 \\
\sum_{n=0}^{N} \sum_{i=1}^{C} z_{n, u}^{(k)}(i, j) & \leq B_{u}^{(k)}(j) \quad \forall k, u, j \\
D_{n, u}^{(k)}(i) D_{n, u^{\prime}}^{\left(k^{\prime}\right)}(i) & =0 \quad \forall n, i, \text { and }(k, u) \neq\left(k^{\prime}, u^{\prime}\right) \\
\sum_{i} D_{n, u}^{(k)}(i) & \leq \eta_{u}^{k} \quad \forall u, k, n \\
D_{n, u}^{(k)}(i) & =0 \quad \forall i, k, n>N_{k} \\
z_{n, u}^{(k)}(i, j) & \geq 0 \quad \forall n, u, k, i, j \\
z_{n, u}^{(k)}(i, j) & =0 \quad \forall\{j: j>(i-1) L+s\}, u, n, k \\
Z_{n, i} & \in \mathcal{Z}_{n} \triangleq\left\{0, Y_{n}\right\} \quad \forall i, n
\end{aligned}
$$

In the above formulation, Constraints (4), (5), (6) and (14) ensure that what is fetched for layer $n$ of chunk $i$ over all links and times is either zero or $Y_{n}$. Constraint (7) imposes the decoding constraint, i.e., it ensures that the $n^{\text {th }}$ layer of any chunk cannot be fetched if the lower layer is not fetched. Constraint (8) imposes the bandwidth constraint of all links at each time slot $j$. Constraint (9) enforces that a chunk's layer can be fetched only over one link. Constraint (10) imposes that the maximum amount of data fetched by link $L_{k, u}$ is $\eta_{u}^{k}$. Constraint (11) enforces that user $u$ at the $k^{t h}$ set cannot be used to fetch layers higher than $N_{k}$. Constraint (12) imposes the non-negativity of the download of a chunk and (13) imposes the deadline constraint since chunk $i \in\{1, \cdots, C\}$ cannot be fetched after its deadline (deadline $(i)=(i-1) L+s)$.

The problem defined in (3)-(14) has integer constraints and a non-convex constraint, i.e., Equation (9). Integer-constrained problems are in the class of discrete optimization, which are known to be NP hard in general [38].

\section{GroupCast Algorithm for Skip-Based STREAMING}

In this section, we describe the proposed algorithm for skip-based streaming. To develop the algorithm, we consider the offline algorithm, i.e., the bandwidth is perfectly known for the entire period of the video. We first assume one set of users, i.e., $K=1$, where there is no preference among different users. We refer to this algorithm as Offline No-Pref GroupCast. This algorithm is then extended to the general case in which users can belong to different sets with different priority levels. We refer to this algorithm as Offline Pref GroupCast. Finally, we propose an online algorithm Online Pref/No-Pref GroupCast in which more practical assumptions are considered such as short bandwidth prediction with prediction error and finite buffer size.

\section{A. Offline No-Pref GroupCast Algorithm}

We now describe Offline No-Pref GroupCast algorithm. For illustration of the algorithm, we have included an example in Supplementary Material. In Offline No-Pref GroupCast, we assume that there is only one set that contains $U$ users. The Offline No-Pref GroupCast algorithm, listed in Algorithm 1, processes the layers sequentially according to their order (Line 4). The first layer to be considered is the base layer where the algorithm initially finds the cumulative bandwidth of every second $j$ and user $u\left(R^{(u)}(j)\right)$ (Line 5). Then, it makes the decision for the base layers of all the chunks, i.e., which chunks to be skipped and which to be fetched. Moreover, the algorithm decides which user is used to fetch the base layer of every chunk that has been decided to be fetched. The algorithm performs a forward scan to find the maximum number of base layers that can be fetched before the deadline of each chunk. The maximum number of base layers that can be fetched before the deadline of the $i^{\text {th }}$ chunk is: $V_{0, i}=$ $\sum_{u=1}^{(U)}\left\lfloor\min \left(\frac{\eta_{u}}{y_{0}}, \frac{R^{u}(\operatorname{deadline}(i))}{y_{0}}\right)\right\rfloor($ Line 6). Let $\operatorname{skip}(i)$ be the total number of skips before the deadline of the $i^{\text {th }}$ chunk. Therefore, if $V_{0, i}$ is less than $i-\operatorname{skip}(i-1)$ at the deadline of the $i^{\text {th }}$ chunk, there must be a skip (or skips), where the total number of skips from the start until the deadline of the $i^{\text {th }}$ chunk is equal to $\operatorname{skip}(i)=\operatorname{skip}(i-1)+1$ (Lines 9-11). If there are $A$ skips, then the algorithm will always skip the first $A$ chunks since they are the closest to their deadlines. Thus, skipping them will result in a bandwidth that can be used by the remaining chunks to increase their quality. This choice maximizes the total available bandwidth for the later chunks. Note that the number of skips could increase if the maximum contribution of all users is used before downloading all chunks.

In the second step, the algorithm performs a backward scan per chunk (Lines 15-24) starting from the first chunk that 
was decided to be fetched by calling the Backward Algorithm (Line 20, Algorithm 2). The backward algorithm simulates fetching every chunk $i$ starting from its deadline and by every user that has $\min \left(R^{(u)}(\right.$ deadline $\left.(i)), \eta_{u}^{k}\right) \geq Y_{0}$ (Lines 16, 18, and 20).

Before we describe the details of the second step, we first define some necessary parameters. Let $\alpha_{i}^{n}(u)$ be the amount of bandwidth used to fetch the layer $n$ of chunk $i$ by user $u$ before the deadline of the $(i-1)^{t h}$ chunk. Let $\zeta_{i}^{n}(u)$ be the cost of fetching the layer $n$ of the $i^{\text {th }}$ chunk by user $u$, and $\zeta_{i}^{n}(u)$ can be found as follows.

$$
\zeta_{i}^{n}(u)= \begin{cases}\alpha_{i}^{n}(u), & \text { if } Y_{n} \text { can be fetched by user } u \\ \infty, & \text { otherwise }\end{cases}
$$

Now we describe the second step of the algorithm. The algorithm performs a backward scan per chunk starting from the first chunk by calling Algorithm 2 (line 20). The backward scan computes the cost of fetching each chunk by each user, i.e., $\zeta_{i}^{n}(u)$. The user that minimizes the cost is chosen to fetch the base layer of the $i^{t h}$ chunk. Note that the link over which the $i^{t h}$ chunk will be fetched is the one that gives the maximum amount of total available bandwidth over all links before the deadline of $(i-1)^{t h}$ chunk. To illustrate, assume that there are 2 users. Consider that fetching the $i^{\text {th }}$ chunk by user 1 results in using $x$ amount of the bandwidth before the deadline of the $(i-1)^{t h}$ chunk, while fetching the $i^{\text {th }}$ chunk by user 2 results in using $y$ amount of the bandwidth before the deadline of the $i-1^{t h}$ chunk. Then, the first user is chosen to fetch the $i^{t h}$ chunk only if $x<y$. The objective of this decision is to save the bandwidth of looming chunks as much as possible. This will help consequent chunks to be fetched at higher layers.

Enhancement Layer Modifications: The algorithm proceeds by performing a forward-backward scan for each enhancement layer in order from lowest to highest quality. The bandwidth is then modified to be the remaining after excluding the reserved bandwidth to fetch lower layers (Line 6 in Algorithm 2). Note that the $n^{\text {th }}$ layer of a chunk is not considered if its $n-1^{\text {th }}$ layer is not decided to be fetched (Line 17 in Algorithm 1).

Downloading Chunks: During the actual fetching of the chunks, each link fetches the layers in order of the chunks. In other words, layer $n$ of the $i^{t h}$ chunk is fetched before layer $m$ of the $j^{t h}$ chunk if and only if $i<j$. Moreover, for the same chunk, the layers are fetched according to their order. For example, the base layer is downloaded first, then enhancement layer 1, and so on.

Complexity Analysis: The algorithm sequentially decides each layer. For each layer $n$, the algorithm first finds $R^{(u)}(j)$, the cumulative bandwidth of each link $u$ and time slot $j$, which is linear with respect to each link. Thus, it has a run-time complexity of $O(U \cdot$ deadline $(C))$. Then, it performs a forward scan that has a run-time complexity of $O(U \cdot$ deadline $(C))$. Finally, a backward scan on each link at each time. Since the complexity of the backward algorithm is linear in $C$ with respect to each link, the run-time complexity for fetching one
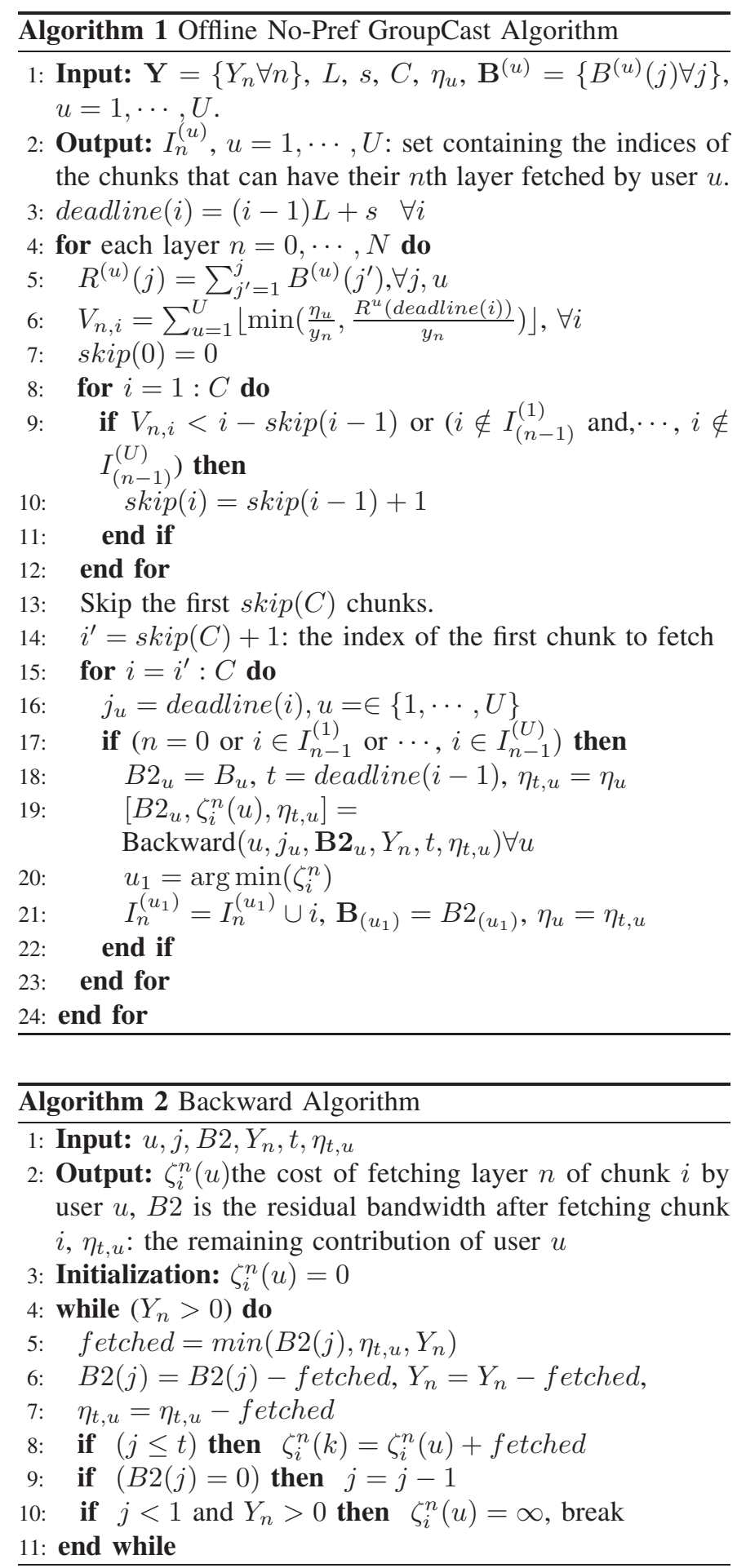

layer is $O\left(U \cdot C^{2}\right)$. Thus, the overall run-time complexity is $O\left(U \cdot N \cdot C^{2}\right)$.

We can show that this algorithm is optimal in two cases: when all chunks are encoded in single layer only, and when the video is encoded into multiple layers but there is one user only. The latter case is obtained by an adaptation of the results in [26].

Even though we do not show the optimality of No-Pref GroupCast in general, we note that this algorithm minimizes the number of skips of the $n^{t h}$ layer given the decisions of all 
lower layers. This result is formally expressed in the following theorem.

Theorem 1: Given size decisions up to $n-1^{\text {th }}$ layer $\left(Z_{0, i}, \cdots, Z_{n-1, i}\right.$, for all $\left.i\right)$, remaining bandwidth, and deadline $(i)$ for every chunk $i$, the proposed No-Pref GroupCast algorithm achieves the minimum number of $n^{\text {th }}$ layer skips (or obtains the maximum number of chunks at layer $n$ ) as compared to any feasible algorithm which fetches the same layers of every chunk up to layer $n-1$.

Proof: Proof is provided in Supplementary Material.

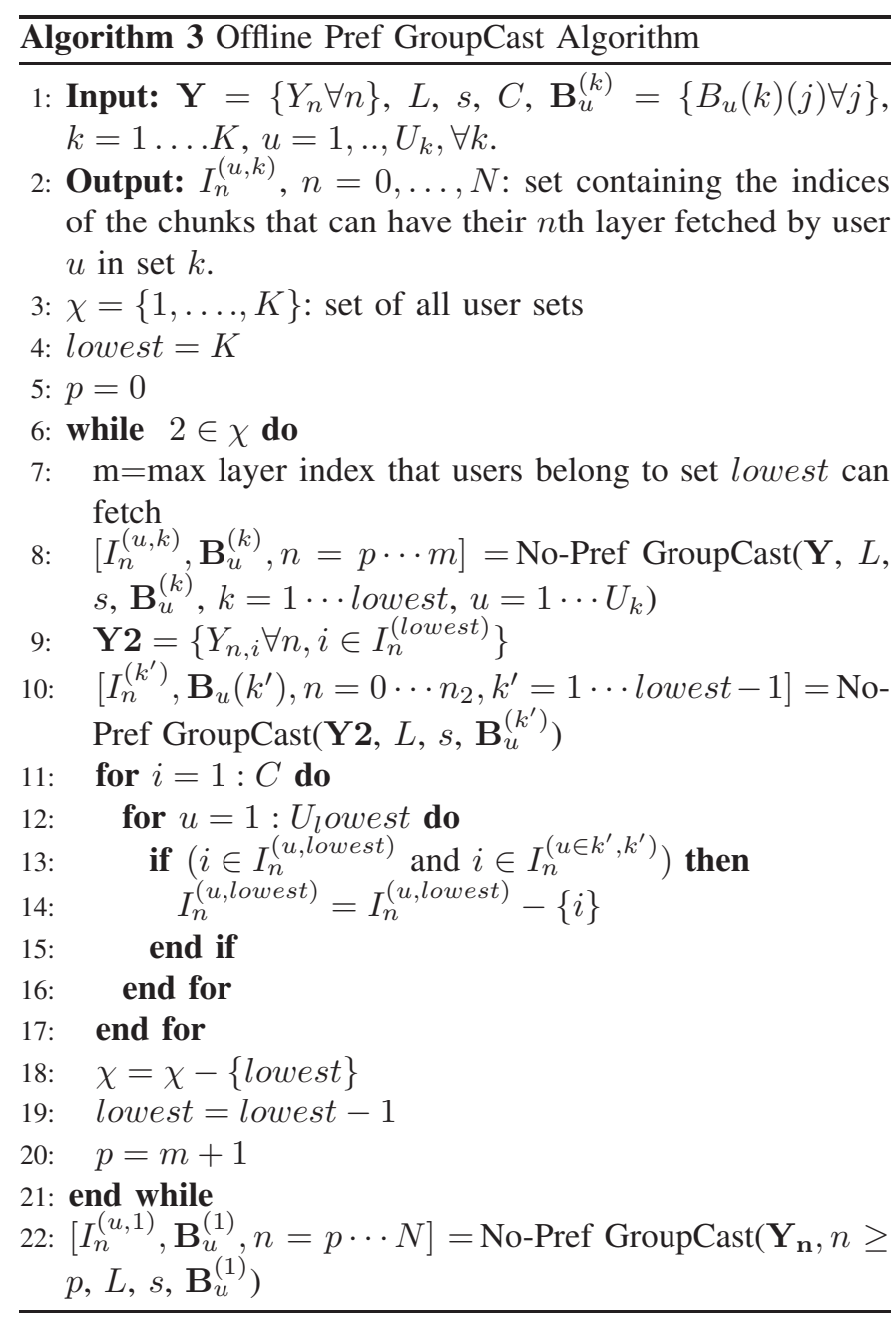

\section{B. Offline Pref-GroupCast Algorithm}

We now describe Pref GroupCast algorithm. For illustration of the algorithm, we have included an example in Supplementary Material. In this section, we consider $K$ sets of users. Users belong to the first set, i.e., users 1 to $u^{\prime}$, have an unlimited data plan and thus can contribute as much as their bandwidths allow. On the other hand, the remaining users, i.e., users $u^{\prime}+1$ to $U$ have different preferences and thus belong to different sets. For example, some users can contribute in fetching up to the $n^{\text {th }}$ enhancement layer, while other users can only help in avoiding skips due to their limited plans. The latter group belong to the set numbered $K$ since sets are ordered according their priority levels. The proposed Offline Pref GroupCast algorithm is listed in Algorithm 3.
The first step in the algorithm is calling No-Pref GroupCast algorithm considering the lowest set of layers that all users can fetch (Line 7-8). We denote these layers by layers 0 to $\mathrm{m}$. The objective of the first call of Offline No Pref-GroupCast is to find the maximum layer less than or equal to $m$ that can be fetched for each chunk. The initial fetching policy obtained by this run is not final since the users preferences have not been taken into consideration. Recall that the lower priority links will not be used to fetch even the the lowest set of layers, i.e., $0, \cdots, m$, if all higher priority links can do so. In the second step, No-Pref GroupCast is executed again for layers 0 to $m$ that were initially decided to be fetched by the users belong to the lowest priority set (set $K$ ) (Lines 9-10). The objective of the second call of No-Pref GroupCast is to move as much as possible of these layers to users belong to the higher priority sets. In the next step, the lowest priority set among the remaining sets is excluded (Line 18), and the same process is repeated. At the last step of the algorithm, No-Pref GroupCast Algorithm is run for the remaining layers considering only the users belonging to the highest priority set (set 1).

\section{Online Adaptations for GroupCast}

For the algorithms described in Sections V-A and V-B, we assume a perfect bandwidth prediction and an unlimited client buffer capacity. In practice, however, bandwidth prediction is not perfect and client's buffer is limited. In fact, even if the buffer is unlimited the video content itself may not be available for a few chunks ahead. Therefore, the buffer constraint is considered to account for both scenarios, i.e., limited client buffer and the availability of future chunks. In this section, we use an online algorithm that obtains bandwidth prediction for a window of size $W$ chunks ahead for each user. Accordingly, the decisions is made based on the prediction. The $W$ chunks start from the $i^{\text {th }}$ chunk that has a current time $\delta$ higher than its deadline, i.e., deadline $(i)=$ current time $+\delta$. Typically, $\delta$ is in the range of $1-2$ seconds. We set $\delta$ as a margin to avoid making a decision for a chunk at its deadline and end up not having its decided layers fully downloaded by its deadline due to prediction error.

There are multiple ways to obtain the bandwidth prediction. Our approach is to use the harmonic mean of the download time of the past $\beta$ layers to predict the future bandwidth [8]. The decisions are re-computed for the chunks that have not yet reached their deadlines periodically every $\alpha$ seconds. Typically, we set $\alpha$ to be $2-3$ seconds. To account for the buffer, we assume that $W L+s$ is no more than the buffer length.

For the next $W$ chunks, the algorithms in Sections V-A and V-B are run to find the quality using the predicted bandwidth. These $W$ chunks can then be fetched according to the decision generated by the algorithms. After $\alpha$ seconds, the optimization problem is re-run, and the decisions are re-considered and updated. The fetching policy is updated on-the-fly without interrupting the download of the current set of layers.

Since the maximum contributions are defined for the whole period of the video, the controller is required to efficiently 
use the maximum contribution per user over the entire duration of the video. In essence, at each time $c \alpha$, where $c \in\{1,2, \ldots\}$, the controller decides the maximum contribution of user $u$ for the current $W$ chunks. Let $\eta_{u}$ be the maximum contribution of user $u$ for the entire video duration of $T$, i.e., $T=(i-1) L+$ $s+d(C)$. Further, let $f_{u, c}$ be the amount of content fetched by user $u$ before the time $c \alpha$. Then, the maximum contribution of user $u$ for the window starting at $c \alpha$, denoted as $\tilde{\eta}_{u, c}$, is as follows.

$$
\tilde{\eta}_{u, c}=\frac{\min (W L+c \alpha L, T)}{T} \cdot \eta_{u}-f_{u, c} .
$$

Accordingly, the maximum contribution per user is fairly distributed over time. In other words, users who contributed less than the assigned maximum contribution in the previous windows contribute more in the current window. The initial contribution is set to $\frac{W L}{T} \cdot \eta_{u}$ in the first window for all users.

\section{No Skip Based Streaming Algorithm}

In no-skip streaming, the video player stalls the video and continues downloading the chunk instead of skipping it when the deadline of the chunk is missed. In this section, the objective is to maximize the average quality while minimizing the stall duration. Accordingly, the new objective function is slightly different from Equation (3) because skipping the base layer is not allowed. However, higher layers can be skipped. All constraints are the same as skip based optimization problem except that we add a new constraint, i.e., Constraint (22), to enforce that $Z_{0, i}$ to be equal to the size of $\mathrm{BL}$ of the $i^{t h}$ chunk $\forall i$. Note that $Z_{0, i}$ cannot be zero because we do not allow skipping base layers. Moreover, the deadline of any chunk $i$ is a function of the total stall from the start of the playback until the playback time of the $i^{\text {th }}$ chunk, i.e., deadline $(i)=(i-1) L+s+d(i)$. We define the total stall duration from the start until the play-time of the $i^{\text {th }}$ chunk as $d(i)$. Therefore, the deadline of the $i^{t h}$ chunk is $(i-1) L+s+d(i)$. The objective function is thus given as:

$$
\sum_{k=1}^{K} \sum_{n=0}^{N} \lambda_{n}^{(k)} G_{n}^{(k)}-\mu d(C)
$$

where the weight for the stall duration is chosen such that $\mu \gg \lambda_{0}^{1}$, because users tend to prefer not running into re-buffering over playing the video in better quality. This is a multi-objective optimization problem with quality and stalls as the two objectives, and is formulated as follows.

$$
\text { Maximize :(17) }
$$

subject to (4), (6), (7), (8), (9), (10), (11), (12), (14)

$$
\begin{aligned}
D_{n, u}^{(k)}(i) & =\sum_{j=1}^{(i-1)} z_{n, u}^{(k)}(i, j) \quad \forall i, n, k, u \\
z_{n, u}^{(k)}(i, j) & =0 \quad \forall\{j: j>(i-1) L+s+d(i)\}, \forall u, n, k \\
d(i) & \geq d(i-1) \geq 0 \quad \forall i>1 \\
Z_{0, i} & =Y_{0}, \quad \forall i
\end{aligned}
$$

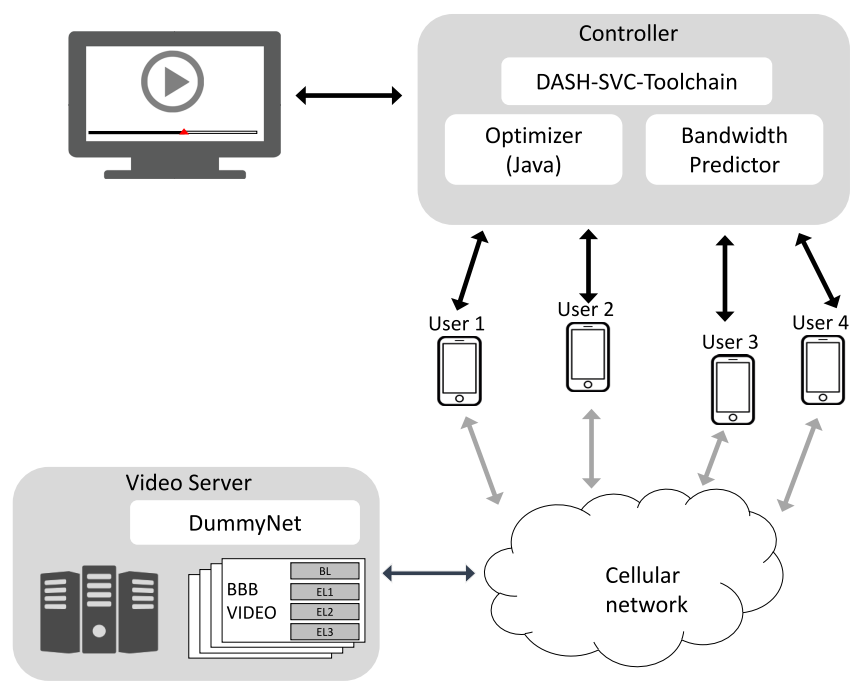

Fig. 2. System setup of the emulated experiment.

As compared to skip based streaming, we do not allow base layer skips as specified by Constraint (22). We note that this problem can be solved using an algorithm similar to the skip-based streaming. One difference as compared to the skip version is that the first step of the no-skip algorithm is to determine the minimum stall time such that all chunks are fetched at least at base layer quality since that is the first priority. Therefore, the forward scan of the base layer objective is not to find the minimum number of skips. The No-PrefGroupCast algorithm runs a base layer forward scan that has the objective of checking if all chunks can be fetched at least at the base layer quality with the current startup delay. At the deadline of the $i^{t h}$, if $V_{0, i}<i$, the algorithm increments the deadline of each chunk $\geq i$ by 1 and resumes the forward scan (Lines 11-15). The algorithm does not proceed to the next chunk untill the condition of $V(i) \geq i$ is satisfied. At the end, the algorithm sets the final deadline of every chunk $i$ to be: deadline $(i)=(i-1) L+s+d(C)$. Therefore, the base layer forward scan achieves the minimum stall duration and brings all stalls to the very beginning. Consequently, this will offer more bandwidth to subsequent chunks and can help increase their quality. The detailed steps are described in Algorithm 5.

The rest of the algorithm is equivalent to the skip version since skips are not allowed only for base layers, i.e., higher layers can be skipped. The key difference in the no-skip with compare to the skip version is that the startup delay is decided such that there will be no skips. No-skip version can be considered as a special case of the skip version in which all chunks can be fetched at least at the base layer without skipping an entire chunk. The No-Pref version of No-Skip GroupCast is described in Algorithm 4.

\section{System EVALUATION}

In this section, we describe the implementation and the evaluation of skip and no-skip versions of GroupCast. However, we only report the results of the skip version because the results for both versions are qualitatively similar. 


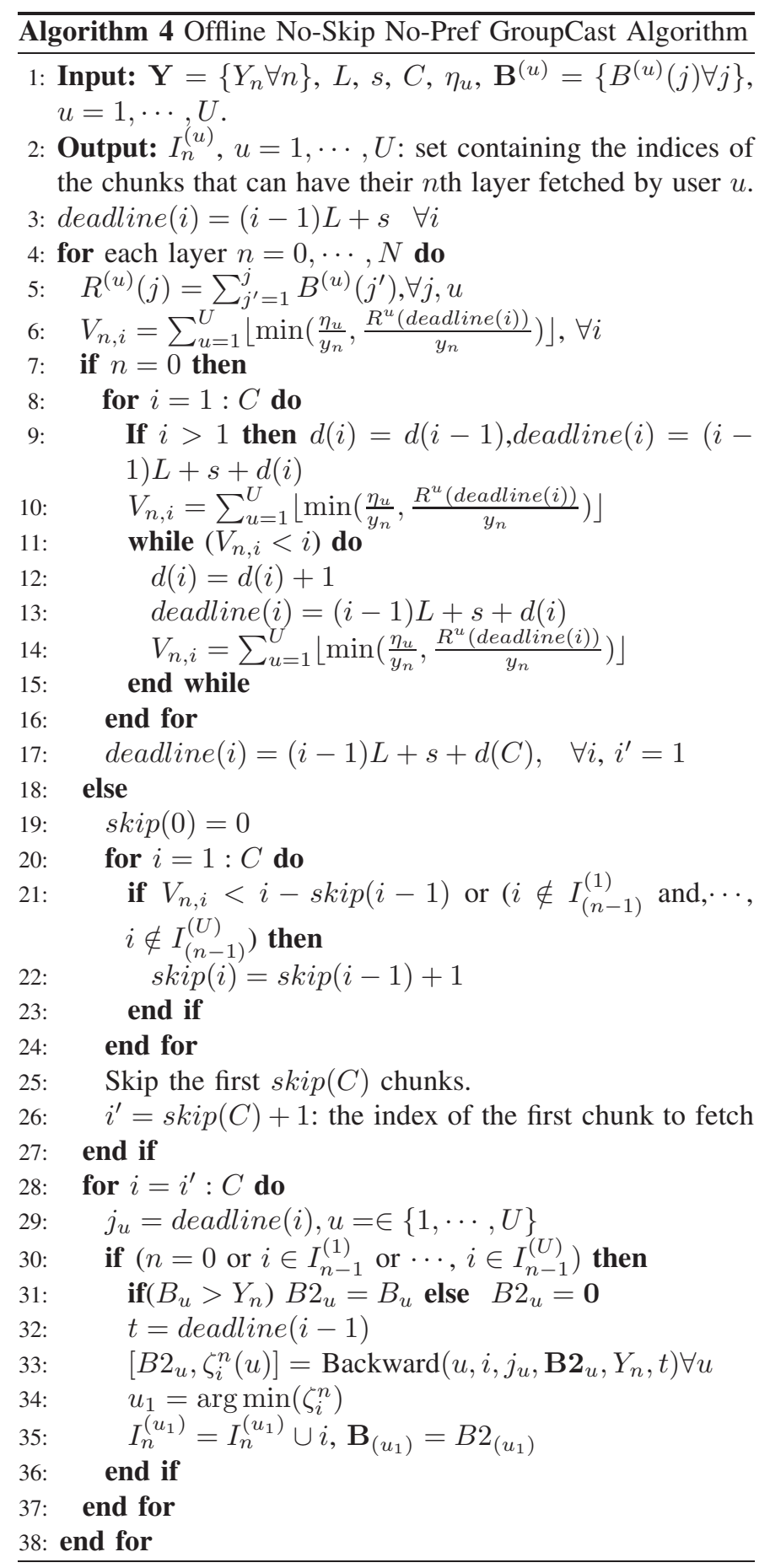

\section{A. Evaluation Parameters}

Implementation Setup. Fig. 2 illustrates the system setup. The implemented system contains three components: (i) the video server, (ii) the controller, and (iii) the clients. The functionalities of the video server include hosting the videos and running the bandwidth shaper. The video server is hosted on a Dell Power Edge R420 with a 6-core Intel E5-2620v3 CPU, 120 GB of RAM, and Ubuntu Server 16.0 LTS OS. The video server runs a Python-based HTTP Server that serves the requests of the clients. Each layer in the video is requested by an HTTP GET request using the layer's URL. We use
TABLE I

SVC ENCODING BITRATES UsEd IN OUR EVALUATION

\begin{tabular}{|c|cccc|}
\hline playback layer & BL & EL1 & EL2 & EL3 \\
\hline Nominal Cumulative Rate (Mbps) & 1.45 & 2.45 & 4.15 & 6.36 \\
\hline
\end{tabular}

the publicly available video "Big Buck Bunny" [23]. The video consists of 299 chunks, i.e., 14315 frames, where each chunk has a duration of 2 seconds (48 frames). We use the quality of SVC scalability mode. The frame rate of this video is $24 \mathrm{fps}$, and the spatial resolution is $1280 \times 720$. The video is encoded into one base layer and three enhancement layers. Table I gives the cumulative nominal rates of each layer, where "BL" and "EL the cumulative $i^{\text {th }}$ enhancement layer rate, respectively. For example, the exact rate of the $i^{t h}$ enhancement layer is equal to $\mathrm{EL}_{i}-\mathrm{EL}_{(i-1)}$, with $\mathrm{EL}_{0}=\mathrm{BL}$. The rate distribution of the different layers of the VBR video is depicted in Fig. 3. For the bandwidth shaping, we use Dummynet [39] tool to limit the outgoing bandwidth from the video server according the collected bandwidth traces. All reported results are based on the 1000 bandwidth traces described below.

The controller contains three components: (i) the Optimizer, (ii) the Bandwidth Predictor, and (iii) the SVC decoder. The functionalities of the controller include providing and orchestrating the communication among users, running the optimizer, sending the decisions to the clients, receiving back the downloaded layers from clients, decoding the received chunks, and playing the video on the display. The optimizer and the bandwidth predictor are implemented in Java. We use the DASH-SVC-Toolchain to decode the video and stream the videos to the display screen [23]. The controller can be hosted on any device, e.g., a phone, a laptop, or a single-board computer, that can provide tethered or wireless connections to the clients and the display. In our implementation, we use a MacBook Pro with $2.4 \mathrm{GHz}$ Intel I5 processor and $8 \mathrm{~GB}$ DDR3 RAM as the controller, which communicates with the clients using an 802.11n WiFi link. Such link does not incur communication overhead, i.e., latency, since the clients are within the proximity of the controller. The $802.11 \mathrm{n}$ network can operate with a net data rate of at least $100 \mathrm{Mbit} / \mathrm{s}$ [40], which is sufficient to serve the users in our setup without being a bottleneck.

The functionalities of the Clients include receiving the fetching policy from the controller, sending the HTTP GET request for each assigned layer to the Video server, and streaming the downloaded layers to the controller. Note that the clients do not decode the downloaded SVC chunks. The client application is implemented in Java and deployed as a mobile phone application. We use four phones as clients, each running Android 7.0. Each phone has 4G of RAM and 32GB of internal memory. We assume that the only connection to the Internet for the controller is provided by a cellular network (LTE/4G/3G) via the clients.

Bandwidth Traces. We use a public dataset that consists of continuous one-second measurement of throughput for cellular system [19]. The dataset has been divided into 1000 traces, each of six minutes length [7]. The statistics of the dataset are shown in Fig. 4(a-b). We assign 250 bandwidth traces 


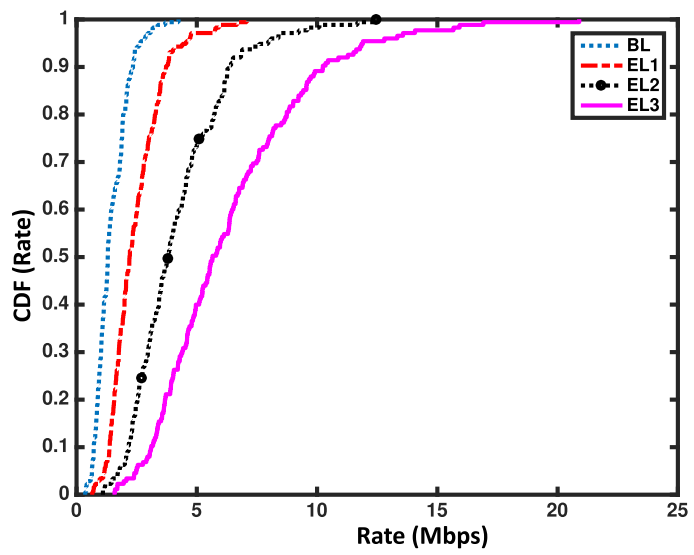

Fig. 3. Rate distribution of the video used in the implementation at different quality levels.

to each user at random. The average throughput across the traces varies from $0.7 \mathrm{Mbps}$ to $2.7 \mathrm{Mbps}$, with a median of 1.6 Mbps. In each trace, the instantaneous throughput is also highly variable, with the average standard deviation across traces being 0.9 Mbps. We only consider the first 175 chunks of the video because that the video length is longer than the provided bandwidth traces.

Experiment Parameters. We considered three experiments scenarios. The first scenario is the no-preference and infinite contribution, i.e., all users contribute equally likely while imposing no maximum contribution to any user. Second scenario is the no-preference and finite contribution, which imposes maximum contribution for all the users. Table II gives the maximum contribution of the four users in $M b$ that are used in the evaluation. We note that the sum of the maximum contributions is $1400 \mathrm{Mb}$, which allows to fetch most of the chunks at the second enhancement layer given that the bandwidth is enough. Notice that the expected size of all the chunks at the second enhancement layer is $4.8 \times 2 \times$ $175=1680 \mathrm{Mb}$. The third scenario is the preference-aware and finite contribution in which users impose the maximum contributions specified in Table II. Moreover, users 3 and 4 are less preferable in the sense that they can only help in avoiding skips, i.e., fetch base layers if the other two users cannot meet the deadlines.

We assume a playback buffer of 10 seconds for all the scenarios considered in the evaluation. In other words, the window size is $W=5$ chunks. Moreover, for all online algorithms, we re-consider the decisions every four seconds, i.e., $\alpha=4 s$, and the parameter $\delta$ is also assumed to be 2 seconds. Finally, the startup delay is 5 seconds.

Bandwidth Prediction. Although that our algorithm can work with any bandwidth prediction technique, we consider a harmonic mean-based prediction. The harmonic mean of the throughput achieved in fetching the last 5 layers by the $i^{\text {th }}$ user is used as a predictor for the bandwidth of that user for the next window of chunks. Since there is no prediction at the beginning, the first 4 chunks will be assigned randomly to the 4 users at base layer quality. Consequently, each user will be fetching one of the first 4 chunks.

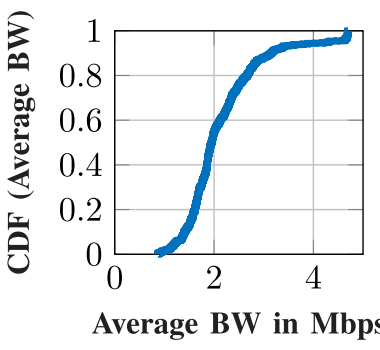

(a)

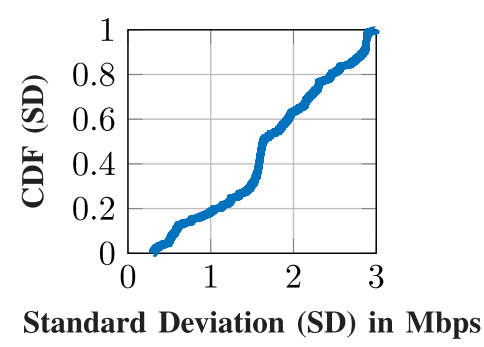

(b)
Fig. 4. Statistics of the two bandwidth traces: (a) mean, and (b) standard deviation of each trace's available bandwidth.

TABLE II

Max Contributions Used in OUR Evaluation

\begin{tabular}{|c|cccc|}
\hline User No. & 1 & 2 & 3 & 4 \\
\hline Max Contribution (Mb) & 672 & 504 & 336 & 168 \\
\hline
\end{tabular}

Comparison Baselines. The proposed online algorithm is compared with the following two strategies based on round robin assignment of layers to users. To achieve fair comparison with the proposed algorithms, we consider maximum contribution per window in the round robin assignment. In particular, a layer is skipped by the user if his residual contribution limit in the window is not enough to fetch that layer. We also include the offline algorithm to show the relative performance of the compared algorithms to our offline scenario, which has perfect knowledge of the bandwidth for the entire period of the video.

Buffer-Based Cooperative Streaming Approach (BB). BB adjusts the streaming quality based on the playback buffer occupancy. Specifically, the quality follows the same strategy of BBA algorithm [15] in making the quality decision. The decision depends on two conditions. First, if the buffer occupancy is lower (higher) than the lower (higher) threshold, then chunks are fetched at the lowest (highest) quality. Second, if the buffer occupancy lies in between the two thresholds, then the buffer rate relationship is determined by a linear function between the two thresholds. We use $4 \mathrm{~s}$ and $10 \mathrm{~s}$ as the lower and higher thresholds on the buffer length, respectively.

Once the decision is obtained, the different layers of the next window of chunks are assigned to users 1, 2, 3, and 4 using round robin strategy for the no-preference scenario. If the maximum contribution of the current window for any of the users does not allow to fetch the base layers, then the next users in the round is chosen. The preference scenario works the same except that if the decided quality is higher than the base layer quality and the maximum contribution of the preferred links allow to fetch base layers, then the enhancement layers are not assigned to the less preferred users. As a result, the less preferred users do not fetch beyond the base layer. If fetching any layer causes to violate the maximum contribution of this window for all users, then that layer is skipped. The process is repeated every $\alpha$ seconds to decide the fetching policy of the next $W$ chunks similar to GroupCast algorithm.

Prediction Based Cooperative Streaming Approach (PB). $\mathrm{PB}$ uses the harmonic mean to predict the bandwidth for the 

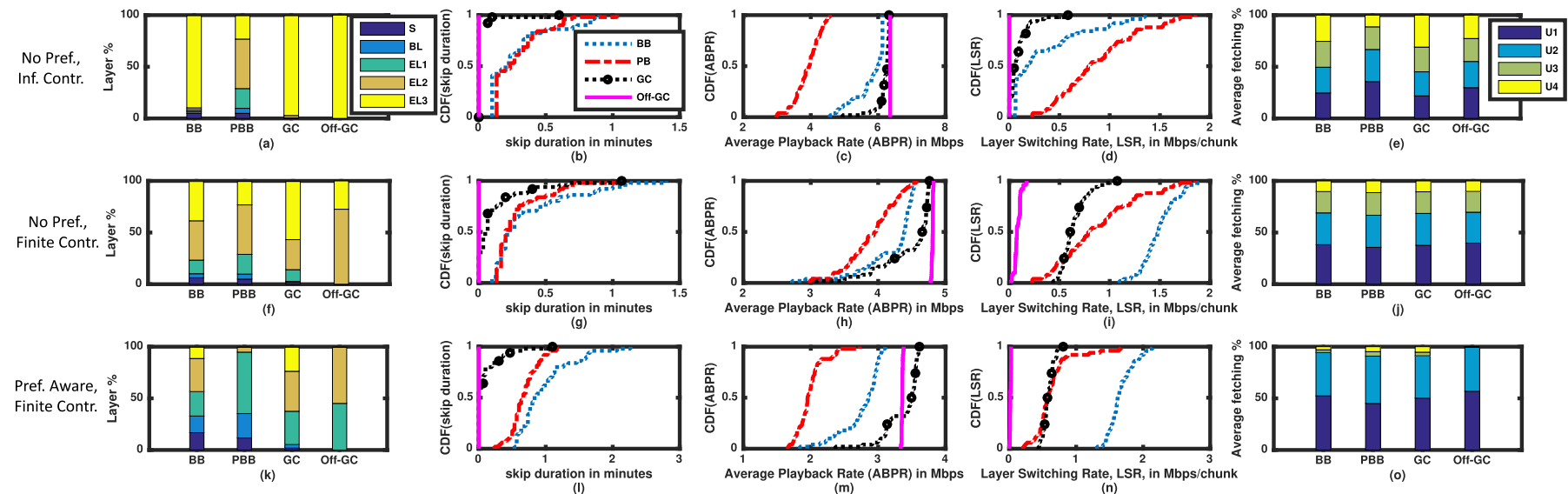

Fig. 5. Comparing streaming algorithms: (a,b,c,d,e) are layer percentage, CDF of the skip duration, CDF of the average playback Rate, APBR, CDF of the layer switching rate, and fetching percentage per user for the no-preference infinite contribution scenario, (f,g,h,i,j) and (k,1,m,n,o) show the same thing for no-preference finite contribution and preference-aware scenario, respectively. Note that legends for the sets (a,f,k), (b,c,d,g,h,i,l,m,n), and (e,j,o) are the same, and are thus not repeated in the subfigures.

TABLE III

Skip Percentage and Average Playback Rate Comparison

\begin{tabular}{|c|c|c|c|c|}
\hline \multicolumn{5}{|c|}{ No Preference, Infinite Contribution } \\
\hline Algorithm & $\overline{\mathrm{BB}}$ & $\mathrm{PB}$ & GroupCast & Off-GroupCast \\
\hline$\%$ of Skips & 4.89 & 3.5 & 0.28 & 0 \\
\hline APBR(Mbps) & 5.77 & 4.0 & 6.18 & 6.35 \\
\hline \multicolumn{5}{|c|}{ No Preference, Finite Contribution } \\
\hline Algorithm & $\mathrm{BB}$ & $\overline{\mathrm{PB}}$ & GroupCast & Off-GroupCast \\
\hline$\%$ of Skips & 6.21 & 4.89 & 2.08 & 0 \\
\hline APBR(Mbps) & 4.20 & 3.90 & 4.46 & 4.80 \\
\hline \multicolumn{5}{|c|}{ Preference-aware, Finite Contribution } \\
\hline Algorithm & $\mathrm{BB}$ & PB & GroupCast & Off-GroupCast \\
\hline$\%$ of Skips & 16.75 & 11.68 & 1.80 & 0 \\
\hline APBR(Mbps) & 2.75 & 2.00 & 3.37 & 3.35 \\
\hline
\end{tabular}

next window of chunks. In particular, PB computes the sum of the predicted bandwidths for all users for the no-preference scenario, and for users 1 and 2 for the preference scenario. Then, it considers $90 \%$ of the computed value as the predicted bandwidth. The closest quality level that is less than the value of the predicted bandwidth is used as the quality decision of all chunks of this window. The decided layers to be fetched are distributed in round robin strategy as described for the BB algorithm.

\section{B. Evaluation Results}

In this subsection, we compare the performance of the GroupCast algorithm with the baseline algorithms described in Section VII-A. We denote the offline fetching policy by Off-GroupCast, where the bandwidth is predicted perfectly and the decisions of all chunks are calculated in a single run of the optimization problem. The maximum contributions are constrained as given in Table II.

The results are illustrated in Table III and Fig. 5. From the results, Off-GroupCast can fetch all chunks at least at the base layer quality without running into skips in all three scenarios. Moreover, we observe that GroupCast achieves the minimum number of skips and the highest playback average among all online algorithms. GroupCast significantly outperforms BB and PB. For example, over all the traces, GroupCast runs in only $0.28 \%, 2.08 \%$, and $1.80 \%$ of skips in the no-preference infinite contribution, no-preference finite contribution, and preference-aware finite contribution, respectively. On the other hand, $\mathrm{BB}$ runs into $4.89 \%, 6.21 \%$, and $16.75 \%$ skips, while PB runs into $3.1 \%, 4.89 \%$, and $11.68 \%$ skips for same respective scenarios.

Fig. 5-(a, f, k) show the layer percentage for each scenario. Fig. 5-a illustrates that there is an assignment policy such that all chunks can be fetched at the highest quality. Moreover, we see that even though GroupCast uses short prediction window with prediction errors, it still achieves the closest performance to the offline algorithm which has the perfect knowledge of the future bandwidth. This is because that we use the sliding based window in which decisions are reconsidered every $\alpha$ seconds.

Fig. 5-(f) demonstrates that the quality drops when the maximum contribution constraint is imposed. According to the maximum contribution values are imposed in Table III, we expect that most of the chunks will be fetched at the $2^{\text {nd }}$ enhancement layer quality, which is also reflected by the off-GroupCast algorithm in Fig. 5-f. We also note that GroupCast is the best algorithm to adjust to the imposed maximum contribution constraints among online algorithms. However, since the online algorithm only has local information per window every $\alpha$ seconds, we observe that it runs into fetching more chunks at the highest quality at the cost of running into subsequent skips.

Fig. 5-(k) shows the results of preference-aware with finite contribution in which users 3 and 4 can only help in avoiding skips. The figure shows that Off-GroupCast can ideally fetch all chunks at either E1 or E2 quality levels. We note similar observations in the no-preference finite contribution scenario, i.e., GroupCast significantly outperform the round robin strategies in avoiding skips and fetch more chunks at higher quality. Moreover, we observe that because of the short prediction of the online GroupCast, it fetches more chunks at the highest quality levels at the cost of skipping some other chunks. Therefore, GroupCat achieves higher average than Off-GroupCast at the cost of more skips, which further degrades the QoE according to our formulation. 
Fig. 5-(b, g, f) and Fig. 5-(c, h, m) show the CDF of the skip percentage and the average playback rates of all of the algorithms over all of the bandwidth traces, respectively. We clearly see that GroupCast achieves the minimum number of skips and the highest average playback rate almost in every single bandwidth trace which reflects the adaptability of GroupCast to different bandwidth regimes and oscillations.

Fig. 5-(d) plots the distribution of the layer switching rate (LSR) for the all bandwidth traces. The LSR of a video is defined as $\frac{1}{C} \sum_{i=2}^{C}|X(i)-X(i-1)| \cdot \mathbf{1}(\Gamma(i) \neq \Gamma(i-1))$, where $C$ is the number of chunks and $\Gamma(i)$ is the highest layer fetched for the $i^{t h}$ chunk. Thus, if two neighboring chunks are fetched at the same layer, then the layer switching is zero even if the layers have different sizes. In other words, we only account for size difference in jumping from one layer to another. As illustrated by the figure, GroupCast achieves a significantly lower LSR as compared to $\mathrm{BB}$ and $\mathrm{PB}$. We note that the LSR is lower than $1 \mathrm{Mbps}$ with probability 1 for GroupCast and Off-GroupCast. However, GroupCast has higher LSR than Off-GroupCast because GroupCast has erroneous bandwidth prediction and only makes local window-based decision.

Fig. 5-(e) shows the percentage of the content fetched by each user. According to the settings of preference-aware finite contribution scenario, users 3 and 4 should only be used to avoid skips. Off-GroupCast shows that users 3 and 4 are used to fetch a negligible number of base layers (almost 0). However, we observe that GroupCast uses users 3 and 4 more than required. The reason is due to the local window-based decision and the bandwidth prediction error of GroupCast. As observed in the figure, GroupCast goes up to fetching chunks at E3 quality level and, as a result, affects the quality of subsequent chunks that cannot meet their deadlines by using only the first two users. Nevertheless, GroupCast uses users 3 and 4 more efficiently than $\mathrm{BB}$ and $\mathrm{PB}$ to avoid further skips because that GroupCast incorporates both users 3 and 4 with their imposed constraints in the optimization-based decision.

Supplementary Material further considers impact of varying prediction window size $(W)$, algorithm update frequency $(\alpha)$, number of collaborating users, and chunk duration. We note that increasing $W$ improves skips first because of not being too aggressive, while then hurts performance due to increased prediction errors. Having smaller $\alpha$ gives more frequent updates and improves the performance. As the number of collaborating users increase, the number of skips see a significant reduction. The average playback rate decreases with an increase in chunk size since the longer chunk size is played at the same quality level.

In conclusion, GroupCast algorithm is able to distribute the layers among users efficiently, thus achieving significantly higher QoE as compared to the considered baselines. We observe that incorporating the chunk deadlines into the optimization problem and favoring the subsequent chunks is essential for the success of the algorithm. This is a unique feature in GroupCast that allows to achieve both low skip duration and high playback quality, while favoring lower layers over the higher layers.

\section{CONCLUSIONS}

In this paper, we propose a preference-aware cooperative video streaming algorithm for SVC-encoded videos. We consider both skip and no-skip based streaming. Finding the quality decisions of the video's chunks and the fetching policy of the SVC layers subject to the available bandwidth, chunk deadlines, and cooperation willingness of the different users was formulated as non-convex optimization problem. A novel algorithm was developed to solve the proposed optimization problem. The proposed algorithm has a polynomial run-time complexity. Real implementation on android devices using SVC-encoded video and bandwidth traces from a public dataset reveal the robustness and performance of the proposed algorithm. The results show that the proposed algorithm improves the number of skips/stalls by at least $57 \%$ as compared to the considered baselines while also improving the average quality.

The paper considers streaming using multiple clients, when the video is encoded with a layered code. Extending the algorithm and implementation over MPEG-DASH with byte-range requests is an interesting future direction. Furthermore, the effect of the SVC scalability dimensions (SNR, spatial, temporal) on the performance of the proposed system requires further studies.

\section{REFERENCES}

[1] A. Elgabli and V. Aggarwal, "GroupCast: Preference-aware cooperative video streaming with scalable video coding," in Proc. INFOCOM Workshop, Apr. 2018, pp. 616-621.

[2] Ericsson Mobility Report on the Pulse of the Networked Society, Ericsson, Stockholm, Sweden, Jun. 2016. [Online]. Available: https://www.abc.es/gestordocumental/uploads/internacional/ EMR_June_2016_D5\%201.pdf

[3] X. K. Zou et al., "Can accurate predictions improve video streaming in cellular networks?" in Proc. 16th Int. Workshop Mobile Comput. Syst. Appl., 2015, pp. 57-62.

[4] G. Iosifidis, L. Gao, J. Huang, and L. Tassiulas, "Enabling crowd-sourced mobile Internet access," in Proc. IEEE INFOCOM, Apr./May 2014, pp. 451-459.

[5] Scalable Video Coding. Accessed: Jul. 14, 2017. [Online]. Available: https://goo.gl/I5C9RL

[6] G. Tech et al., "Overview of the multiview and 3D extensions of high efficiency video coding," IEEE Trans. Circuits Syst. Video Technol., vol. 26, no. 1, pp. 35-49, Jan. 2016.

[7] X. Yin, A. Jindal, V. Sekar, and B. Sinopoli, "A control-theoretic approach for dynamic adaptive video streaming over HTTP," in Proc. ACM SIGCOMM, 2015, pp. 325-338.

[8] Y.-C. Chen, D. Towsley, and R. Khalili, "MSPlayer: Multi-source and multi-path video streaming," IEEE J. Sel. Areas Commun., vol. 34, no. 8, pp. 2198-2206, Aug. 2016.

[9] Apple. HTTP Live Streaming. Accessed: Jul. 14, 2017. [Online]. Available: https://goo.gl/6yYWg

[10] Microsoft Smooth Streaming. Accessed: Jul. 14, 2017. [Online]. Available: http://goo.gl/TQHWL

[11] Adobe. HTTP Dynamic Streaming. Accessed: Jul. 14, 2017. [Online]. Available: http://goo.gl/IZWE8d

[12] K. Miller, D. Bethanabhotla, G. Caire, and A. Wolisz, "A controltheoretic approach to adaptive video streaming in dense wireless networks," IEEE Trans. Multimedia, vol. 17, no. 8, pp. 1309-1322, Aug. 2015.

[13] D. Jarnikov and T. Özçelebi, "Client intelligence for adaptive streaming solutions," Signal Process., Image Commun., vol. 26, no. 7, pp. 378-389, 2011.

[14] M. Claeys, S. Latre, J. Famaey, and F. De Turck, "Design and evaluation of a self-learning HTTP adaptive video streaming client," IEEE Commun. Lett., vol. 18, no. 4, pp. 716-719, Apr. 2014. 
[15] T.-Y. Huang, R. Johari, N. McKeown, M. Trunnell, and M. Watson, "A buffer-based approach to rate adaptation: Evidence from a large video streaming service," in Proc. ACM SIGCOMM, 2014, pp. 187-198.

[16] X. Liu et al., "A case for a coordinated Internet video control plane," in Proc. ACM SIGCOMM, 2012, pp. 359-370.

[17] A. Ganjam et al., "C3: Internet-scale control plane for video quality optimization," in Proc. NSDI, 2015, pp. 131-144.

[18] Y. Sun et al., "CS2P: Improving video bitrate selection and adaptation with data-driven throughput prediction," in Proc. ACM SIGCOMM, 2016, pp. 272-285.

[19] H. Riiser, P. Vigmostad, C. Griwodz, and P. Halvorsen, "Commute path bandwidth traces from 3G networks: Analysis and applications," in Proc. 4th ACM Multimedia Syst. Conf., 2013, pp. 114-118.

[20] J. Hao, R. Zimmermann, and H. Ma, "GTube: Geo-predictive video streaming over HTTP in mobile environments," in Proc. ACM MMSys, 2014, pp. 259-270.

[21] A. Elgabli and V. Aggarwal. (2018). "FastScan: Robust low-complexity rate adaptation algorithm for video streaming over HTTP." [Online]. Available: https://arxiv.org/abs/1806.02803

[22] Joint Video Team (JVT) of ISO/IEC MPEG \& ITU-T VCEGg. Accessed: Jul. 2007. [Online]. Available: http://ip.hhi.de/imagecom_G1/ savce/downloads/H.264-MPEG4-AVC-Version8-FinalDraft.pdf

[23] C. Kreuzberger, D. Posch, and H. Hellwagner, "A scalable video coding dataset and toolchain for dynamic adaptive streaming over HTTP," in Proc. ACM MMSys, 2015, pp. 213-218.

[24] T. Andelin, V. Chetty, D. Harbaugh, S. Warnick, and D. Zappala, "Quality selection for dynamic adaptive streaming over HTTP with scalable video coding," in Proc. ACM MMSys, 2012, pp. 149-154.

[25] C. Sieber, T. Hoßfeld, T. Zinner, P. Tran-Gia, and C. Timmerer, "Implementation and user-centric comparison of a novel adaptation logic for DASH with SVC," in Proc. IFIP/IEEE Int. Symp. Integr. Netw. Manage., May 2013, pp. 1318-1323.

[26] A. Elgabli, V. Aggarwal, S. Hao, F. Qian, and S. Sen, "LBP: Robust rate adaptation algorithm for SVC video streaming," IEEE/ACM Trans. Netw., vol. 26, no. 4, pp. 1633-1645, Aug. 2018.

[27] Multipath TCP in the Linux Kernel. Accessed: Jul. 14, 2017. [Online]. Available: http://www.multipath-tcp.org

[28] Q. D. Coninck, M. Baerts, B. Hesmans, and O. Bonaventure, "A first analysis of multipath TCP on smartphones," in Proc. 17th Int. Passive Active Meas. Conf., vol. 17. Cham, Switzerland: Springer, Mar./Apr. 2016, pp. 57-69.

[29] A. Elgabli, K. Liu, and V. Aggarwal, "Optimized preference-aware multi-path video streaming with scalable video coding," IEEE Trans. Mobile Comput., to be published. doi: 10.1109/TMC.2018.2889039.

[30] M. Stiemerling and S. Kiesel, "A system for peer-to-peer video streaming in resource constrained mobile environments," in Proc. 1st ACM Workshop User-Provided Netw., Challenges Opportunities, 2009, pp. 25-30.

[31] L. Keller et al., "MicroCast: Cooperative video streaming on smartphones," in Proc. 10th Int. Conf. Mobile Syst., Appl., Services, 2012, pp. 57-70.

[32] M. Grafl et al., "Scalable media coding enabling content-aware networking," IEEE Multimedia, vol. 20, no. 2, pp. 30-41, Apr./Jun. 2013.

[33] M. Eberhard, T. Szkaliczki, H. Hellwagner, L. Szobonya, and C. Timmerer, "Knapsack problem-based piece-picking algorithms for layered content in peer-to-peer networks," in Proc. ACM Workshop Adv. Video Streaming Techn. Peer-to-Peer Netw. Social Netw., 2010, pp. 71-76

[34] E. Yaacoub, F. Filali, and A. Abu-Dayya, "SVC video streaming over cooperative LTE/802.11p vehicle-to-infrastructure communications," in Proc. World Congr. Comput. Inf. Technol. (WCCIT), Jun. 2013, pp. 1-5.

[35] C.-H. Lee, C.-M. Huang, C.-C. Yang, and T.-H. Wang, "Co-SVC-MDCbased cooperative video streaming over vehicular networks," Comput. J., vol. 55, no. 6, pp. 756-768, 2011.

[36] R. An, Z. Liu, and Y. Ji, "SVC-based cooperative video streaming in highway vehicular networks," in Proc. 31st Int. Conf. Adv. Inf. Netw. Appl. Workshops (WAINA), Mar. 2017, pp. 216-221.
[37] A. Elgabli, M. Felemban, and V. Aggarwal, "GiantClient: Video hotspot for multi-user streaming," IEEE Trans. Circuits Syst. Video Technol., to be published.

[38] G. L. Nemhauser and L. A. Wolsey, "Integer programming and combinatorial optimization," in Constraint Classification for Mixed Integer Programming Formulations, vol. 20. G. L. Nemhauser, M. W. P. Savelsbergh, G. S. Sigismondi, Eds. Chichester, U.K.: Wiley, 1992, pp. 8-12.

[39] The Dummynet Project. Accessed: Jul. 14, 2017. [Online]. Available: http://info.iet.unipi.it/luigi/dummynet/

[40] Y. Xiao, "IEEE 802.11n: Enhancements for higher throughput in wireless LANs," IEEE Wireless Commun., vol. 12, no. 6, pp. 82-91, Dec. 2005.

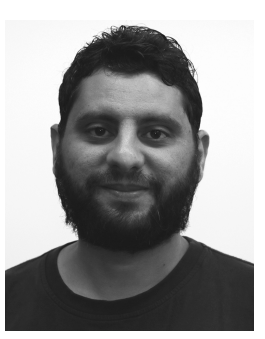

Anis Elgabli received the B.S. degree in electrical and electronic engineering from the University of Tripoli, Tripoli, Libya, in 2004, the M.Eng. degree in electrical and computer engineering from UKM, Kajang, Malaysia, in 2006, and the M.S. and Ph.D. degrees in electrical and computer engineering from Purdue University, IN, USA, in 2015 and 2018, respectively. He joined the Center of Wireless communication, University of Oulu, Oulu, Finland, as a Postdoctoral Researcher, in 2019. His research interest is in applying optimization techniques in networking and communication systems. He was a recipient of the 2018 Infocom Workshop HotPOST Best Paper Award.

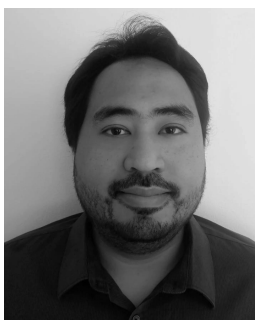

Muhamad Felemban (S'12) received the B.S. degree from KFUPM, Saudi Arabia, in 2008, the M.S. degree from KAUST, Saudi Arabia, in 2011, and the Ph.D. degree from the School of Electrical and Computer Engineering, Purdue University, in 2018. He is currently an Assistant Professor with KFUPM. His research interests include cyber security, data security and privacy, and big data analytics.

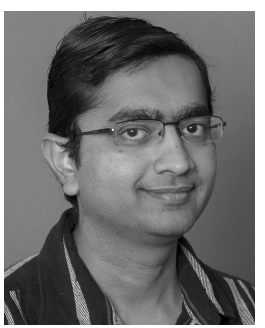

Vaneet Aggarwal (S'08-M'11-SM'15) received the B.Tech. degree from the IIT Kanpur, India, in 2005, and the M.A. and Ph.D. degrees from Princeton University, Princeton, NJ, USA, in 2007 and 2010, respectively, all in electrical engineering.

He was a Senior Member of the Technical Staff Research with AT\&T Labs Research, Bedminster, NJ, USA, from 2010 to 2014. He has been an adjunct Assistant Professor at Columbia University from 2012 to 2014, and the VAJRA Adjunct Professor at IISc Bangalore from 2018 to 2019. He is currently an Associate Professor at Purdue University, West Lafayette, IN, USA. His current research interests are in communications and networking, video streaming, cloud computing, and machine learning. He received Princeton University's Porter Ogden Jacobus Honorific Fellowship in 2009, which is the top honor for graduate students in their final year, the AT\&T Vice President Excellence Award in 2012, the AT\&T Key Contributor Award in 2013, the AT\&T Senior Vice President Excellence Award in 2014, the 2017 Jack Neubauer Memorial Award recognizing the Best Systems Paper published in the IEEE TRANSACTIONS ON VEHICULAR TECHNOLOGY, and the 2018 Infocom Workshop HotPOST Best Paper Award. He is on the Editorial Board of the IEEE TRANSACTIONS ON COMMUNICATIONS and the IEEE TRANSACTIONS ON GREEN COMMUNICATIONS AND NETWORKING. 